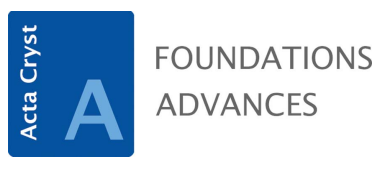

ISSN 2053-2733

Received 15 April 2020

Accepted 21 April 2020

Keywords: embedded nets; isotopy classification; topology.

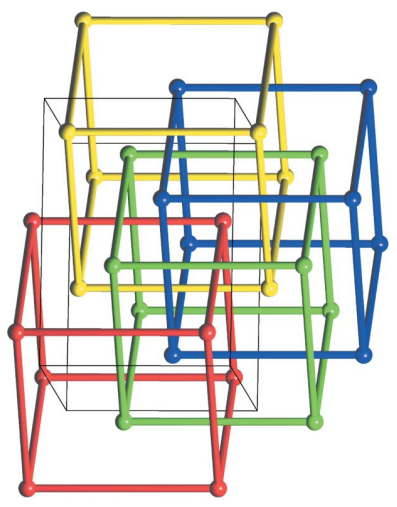

(C) 2020 International Union of Crystallography

\section{Isotopy classification of three-dimensional embedded nets}

\author{
Egon Schulte* \\ Northeastern University, Department of Mathematics, Boston, MA 02115, USA. *Correspondence e-mail: \\ e.schulte@northeastern.edu
}

Nets are important mathematical tools that have been used as models in the analysis and design of crystal structures since the early days of crystallography (Wells, 1977). The notion of a net is extremely versatile and lends itself to major applications in crystal engineering and crystal structure prediction, including metal-organic frameworks (MOFs) and zeolites. Apart from this wide range of applicability, nets and their embeddings are also fascinating mathematical objects in their own right and have significant applications in mathematics itself, including in polyhedral geometry, tiling theory, minimal surfaces and discrete symmetry, to name a few.

The underlying topological structure of a net is provided by an abstract graph $G$, with finite or (usually) countably infinite vertex set and edge set, and in the infinite case equipped with an abstract periodicity structure determined by a free abelian subgroup of the combinatorial automorphism group of $G$. The infinite graphs come to life through their 3-periodic embeddings as periodic bond-node structures $\mathcal{N}$ in an ambient geometric space, which in most applications is ordinary Euclidean 3 -space $\mathbb{R}^{3}$. These embedded nets $\mathcal{N}$ can have several different characteristics. In the embedding, the vertices of $G$ are represented by (usually distinct) points, called the vertices (or nodes) of $\mathcal{N}$, and the edges of $G$ are represented by what are called edges (or bonds) of $\mathcal{N}$, which usually are straight line segments (sometimes with a specified length), possibly allowed to collide, or sometimes are non-self-intersecting paths of various sorts. The entire embedded net $\mathcal{N}$ is required to be invariant under a group of translations $T$ of the ambient space generated by the translations in the directions of the basis vectors from a vector-space basis, called the periodicity basis of $\mathcal{N}$. This translation group $T$ provides a geometric representation of the abstract periodicity structure on the underlying structure graph. In a proper linear 3-periodic net $\mathcal{N}$ in $\mathbb{R}^{3}$, the edges of $\mathcal{N}$ are noncolliding straight line segments joining vertices of $\mathcal{N}$. In applications, the vertices of an embedded net $\mathcal{N}$ may represent spatial positions of atoms or of clusters of atoms, such as single metal ions or secondary building units, and the edges may represent chemical bonds or ligands. A net can represent many compounds.

My own interest in nets relates to discrete geometry and was inspired by the pioneering contributions of Michael O'Keeffe and his collaborators (see, for example, DelgadoFriedrichs et al., 2005). These included Omar Yaghi, who was a co-founder of reticular chemistry with O'Keeffe (for which they were jointly awarded the Gregori Aminoff Prize in Crystallography by The Royal Swedish Academy of Sciences in 2019), and Davide Proserpio, to whom I am grateful for making me aware of the extensive works on crystal nets and related geometric structures in the chemistry literature (and for kindly providing the two figures). Several databases have been created to keep track of the vast amount of information on net structure, including the Reticular Chemistry Structure Resource (RCSR) and ToposPro. The RCSR database was constructed as a tool for the design of new structures of crystalline materials and contains a rich collection of known crystal nets including the most symmetric nets (http://rcsr.net and O'Keeffe et al., 2008) and homogeneous sphere packings (e.g. Fischer et al., 2006, and references therein). While the RCSR database contains information on $3000+$ named nets, ToposPro is a more recent research tool for the geometrical and topological analysis of crystal structures with a database of more than 190000 nets (http://topcryst.com and Blatov et al., 2014). These rapid developments in net theory provide the context for the work by Power et al. (2020). 
The article by Stephen Power, Igor Baburin and Davide Proserpio in this issue of Acta Crystallographica Section A studies isotopy classes of 3-periodic embedded nets in ordinary 3-space. Their work exploits the combinatorial structure of quotient graphs of embedded nets, as well as realizations of quotient graphs as linear graph knots (with linear edges) in the three-dimensional flat torus $\mathbb{T}^{3}$, to obtain periodic isotopy classifications for various kinds of embedded nets. Attractive features of the paper - appreciated by the non-expert - are the detailed introduction and review of the various notions of net-like structures that have been investigated in the literature, from periodic graphs, to linear 3periodic nets, to crystallographic bar-joint frameworks, to string-node nets, as well as providing rigorous definitions and basics for key concepts used in the analysis of nets, from connectedness, to entanglement and self-entanglement, to isotopy equivalence, to quotient graphs, to linear graph knots.

The focus of the article is on the finer classification and enumeration tools for periodic net embeddings beyond the usual classification into isomorphism types. Embedded nets can have multiple connected components, which can have different dimensionality and be intertwined in rather sophisticated ways. The article by Power et al. (2020) mainly concerns the behavior of entangled embedded nets with multiple connected components, as well as of self-entangled connected embedded nets, with regards to continuous deformations that avoid edge collisions or may satisfy some other desirable property (e.g. retaining some translational symmetry across the deformation path as formalized by the notion of periodic isotopy). Little was previously known about the systematic classification of entangled embedded nets into equivalence classes under such deformations.

More specifically, Power et al. (2020) obtain periodic isotopy classifications for various families of embedded nets with small quotient graphs (see Fig. 1 for an example). Quotient graphs of linear periodic embedded nets $\mathcal{N}$ are finite edge-labeled multi-graphs, with integer vectors as edge labels, which capture the action of the underlying translation group $T$ on the vertices and edges of $\mathcal{N}$. Power et al. (2020) enumerate the 25 periodic isotopy classes of linear 3-periodic nets in $\mathbb{R}^{3}$ with adjacency depth 1 (all coordinates of the edge-labeling integer

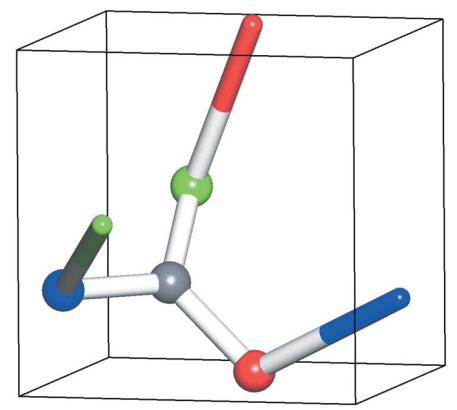

Figure 1

A linear graph knot on the flat 3-torus for the net srs of strontium silicide, $\mathrm{SrSi}_{2}$, similar to an example shown in Power et al. (2020). A linear graph knot is a geometric realization in the flat torus of the labeled quotient graph of a linear periodic embedded net in Euclidean space. Image kindly provided by Davide Proserpio.

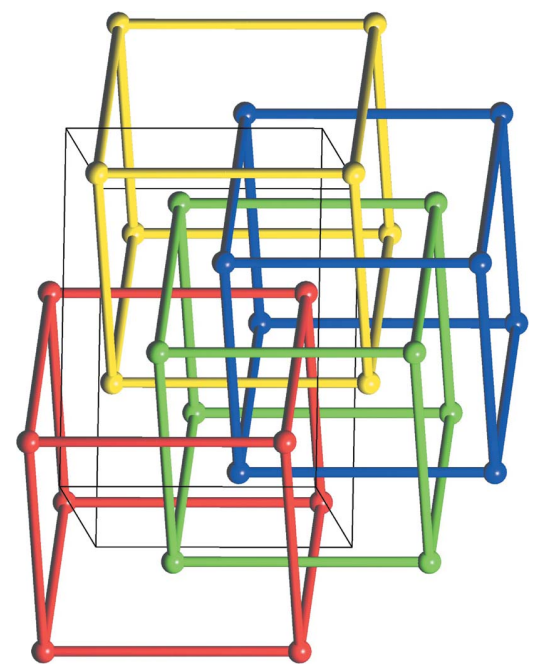

Figure 2

Four translative copies of the primitive cubic lattice pcu, an example of a 'shift-homogeneous' 4-grid in space. Image kindly provided by Davide Proserpio.

vectors have a modulus of at most 1). They also determine the number of periodic isotopy classes for various families of linear 3-periodic nets in $\mathbb{R}^{3}$ whose connected components are embeddings of the net of the primitive cubic lattice pcu, including in particular the family of multigrids important for coordination polymers. Fig. 2 shows an example of a fourfold pcu, a 4-grid.

The article by Power et al. (2020) takes a very significant step in the directions of understanding the fascinating behavior of continuously changing entangled embedded nets in space and of describing the isotopy classes for various important families of embedded nets. Their work features an attractive blend of mathematical techniques from geometry, topology and group theory, brought to bear on difficult questions in crystal chemistry. Classification of crystal nets by isotopy is a challenging endeavor that will attract a lot more attention in the future.

\section{Funding information}

Egon Schulte was supported by the Simons Foundation Award No. 420718.

\section{References}

Blatov, V. A., Shevchenko, A. P. \& Proserpio, D. M. (2014). Cryst. Growth Des. 14, 3576-3586.

Delgado-Friedrichs, O., Foster, M. D., O'Keeffe, M., Proserpio, D. M., Treacy, M. M. J. \& Yaghi, O. M. (2005). J. Solid State Chem. 178, 2533-2554.

Fischer, W., Sowa, H. \& Koch, E. (2006). Acta Cryst. A62, 413-418.

O'Keeffe, M., Peskov, M. A., Ramsden, S. J. \& Yaghi, O. M. (2008). Acc. Chem. Res. 41, 1782-1789.

Power, S. C., Baburin, I. A. \& Proserpio, D. M. (2020). Acta Cryst. A76, 275-301.

Wells, A. F. (1977). Three-Dimensional Nets and Polyhedra. New York: Wiley-Interscience. 